\title{
Hospital mortality in acute coronary syndrome: adjustment of GRACE score by D-dimer enables a more accurate prediction in a prospective cohort study
}

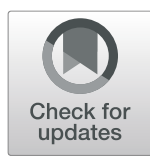

Tongtong Yu, Yundi Jiao, Jia Song, Dongxu He, Jiake Wu, Zhijun Sun and Zhaoqing Sun ${ }^{*}$ (D)

\begin{abstract}
Backgroud: To assess the value of D-dimer and its combination with The Global Registry of Acute Coronary Events (GRACE) score in predicting in-hospital mortality in patients with acute coronary syndrome (ACS) undergoing percutaneous coronary intervention (PCl).

Methods: In 5923 ACS patients undergoing PCl, the role of D-dimer and the added value of D-dimer to GRACE score for predicting in-hospital mortality were tested.

Results: After multivariable adjustment, D-dimer could significantly predict in-hospital mortality. Also, it could significantly improve the prognostic performance of GRACE score (C-statistic: $z=2.269, p=0.023$; IDI: 0.016, $p=0.032$; NRI: $0.291, p=0.035)$.
\end{abstract}

Conclusion: In patients with ACS undergoing PCl, D-dimer was an independent predictor of in-hospital death. It could also improve the prognostic performance of GRACE score.

Keywords: D-dimer, In-hospital mortality, Acute coronary syndrome, GRACE score, Percutaneous coronary intervention

\section{Background}

Acute coronary syndrome (ACS) patients still have a poor prognosis, even receiving timely percutaneous coronary intervention (PCI) and/or adequate antiplatelet drugs [1-4]. Early risk stratification is essential for making clinical decision and evaluating prognosis. D-dimer is a kind of degradation product of fibrin [5]. It is also a biomarker of coagulation state and the form of thrombosis [5]. Elevated D-dimer was associated with vulnerable plaque [6], no-reflow after PCI $[7,8]$ and a larger myocardial injury assessed by cardiac magnetic resonance (CMR) [9]. Many studies also confirmed the association between higher D-dimer and the increased long-term mortality in patients with stable coronary artery disease [10, 11], ACS [12] and ST-segment elevation myocardial infarction (STEMI) $[13,14]$. However, it isn't well known about the

\footnotetext{
* Correspondence: sunzhaoqing@vip.163.com

Department of Cardiology, Shengjing Hospital of China Medical University, Shenyang, Liaoning, People's Republic of China
}

role of D-dimer in predicting in-hospital mortality in ACS patients.

The Global Registry of Acute Coronary Events (GRACE) score is recommended to get in-hospital mortality in ACS patients and can help us make clinical decision and discriminate the patients on high-risk [1-4]. But, GRACE score doesn't include new risk factors, such as Ddimer. Whether adjustment of the GRACE risk score by D-dimer will enable a more accurate prediction is not also defined.

This study tried to confirm whether D-dimer could predict in-hospital mortality, and improve the prognostic performance of the GRACE score in patients with ACS undergoing PCI.

\section{Methods}

\section{Study design and setting}

This study included the consecutive patients with ACS undergoing PCI at a large-scale hospital in Northeast

(c) The Author(s). 2019 Open Access This article is distributed under the terms of the Creative Commons Attribution 4.0 International License (http://creativecommons.org/licenses/by/4.0/), which permits unrestricted use, distribution, and reproduction in any medium, provided you give appropriate credit to the original author(s) and the source, provide a link to the Creative Commons license, and indicate if changes were made. The Creative Commons Public Domain Dedication waiver (http://creativecommons.org/publicdomain/zero/1.0/) applies to the data made available in this article, unless otherwise stated. 
China (Shengjing Hospital of China Medical University, Shenyang, China) from January 1, 2010 to December 31, 2017. The investigators got clinical and procedural data of all cases from electronic medical records and Picture Archiving and Communication Systems. GRACE score was gained as defined previously [1-4]. Venous blood samples were drawn from all cases on admission and measured for D-dimer using latex agglutination assays by an automatic coagulation analyzer (ACL TOP, BECKMAN COULTER, USA) in Shengjing Hospital Core Laboratory. The reference interval of D-dimer was 0$252 \mathrm{ng} / \mathrm{mL}$. In-hospital death was defined previously [15]. Exclusion criteria included (1) use of erythropoietin, oral anticoagulants and thrombolysis (75 cases); (2) severe infections, end-stage liver or renal failure (221 cases); (3) known autoimmune diseases or steroid therapy, known malignancy, recent ischemic or hemorrhagic disease (32 cases); (4) recently undergone surgical or invasive procedures (12 cases); (5) samples collected within $5 \mathrm{~h}$ after use of unfractionated heparin or $12 \mathrm{~h}$ after use of low molecular weight heparin (LMWH) (187 cases); (6) GRACE score data missing (41 cases). At last, 5923 patients with ACS undergoing PCI were included in this study. They were then divided into three groups according to the tertile of D-dimer level (Low D-dimer group: $\leq 88 \mathrm{ng} / \mathrm{mL} \quad[n=1975]$; Intermediate $\mathrm{D}$-dimer group: $89-179 \mathrm{ng} / \mathrm{mL}$ ) [ $n=1974]$; High D-dimer group: $>179 \mathrm{ng} / \mathrm{mL}[n=1974])$. This study complies with the Declaration of Helsinki, and Shengjing Hospital of China Medical University Research Ethics Committee approved the research protocol. Written informed consent was formally obtained from all participants.

\section{Statistical analysis}

Quantitative variables with normal distribution were represented as mean \pm standard deviation (SD) and compared with variance analysis. Quantitative variables without normal distribution were represented as median [interquartile range, IQR] and compared with Kruskal-Wallis $\mathrm{H}$ test. Categorical variables are presented as counts and

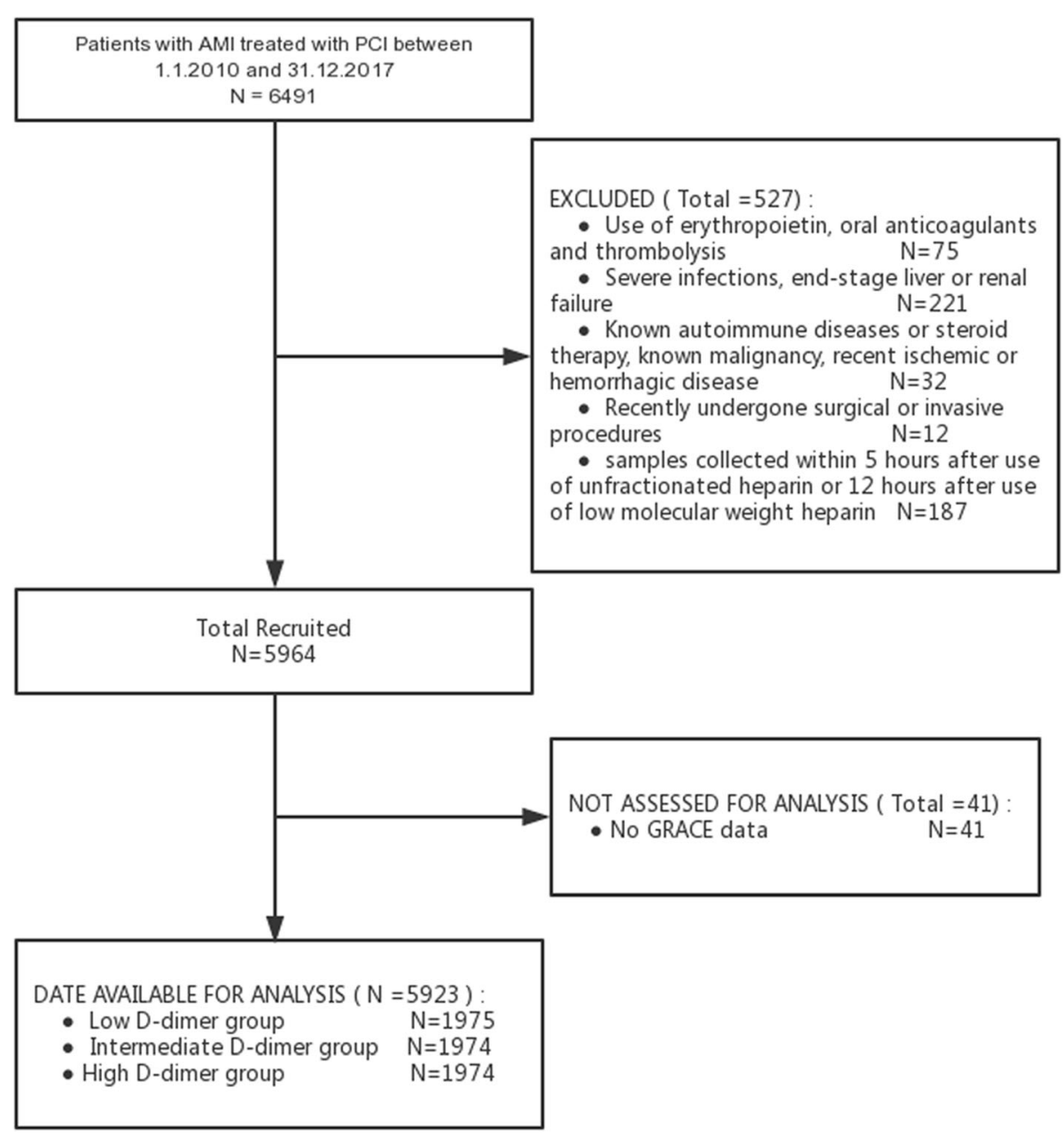

Fig. 1 Flow diagram of participant selection 
proportions (\%) and compared with chi-square test. Logistic univariate regressions were performed to evaluate predictors of mortality of all variables (Additional file 1: Table S1). A multivariate logistic regression model was used to identify independent predictors of mortality. Variables with $p<0.1$ on univariate analysis were entered a multivariate analysis (Additional file 1: Table S1). D-dimer was analyzed as a continuous variable and a categories variable, respectively. Results were reported as odds ratios (ORs) with associated 95\% confidence intervals (CIs). The predictive performance of D-dimer, GRACE score and GRACE score + D-dimer was assessed by indexes of discrimination (C-statistic), calibration (the HosmerLemeshow test, the Nagelkerke- $\mathrm{R}^{2}$ ) and precision (the Brier scores). The C-statistic was compared using a nonparametric test developed by DeLong et al. [16] Each model was entered into a logistic regression model to get the individual risk probability of all-cause death, respectively. The Hosmer-Lemeshow (HL) test and the Nagelkerke- $\mathrm{R}^{2}$ from the regression modeling was used as

Table 1 Baseline Characteristics of the study population, median (IQR), or N (\%), or means \pm SD

\begin{tabular}{|c|c|c|c|c|c|}
\hline Variable & $\begin{array}{l}\text { Overall } \\
(n=5923)\end{array}$ & $\begin{array}{l}\text { Low D-dimer } \\
\text { group }(n=1975)\end{array}$ & $\begin{array}{l}\text { Intermediate D-dimer } \\
\text { group }(n=1974)\end{array}$ & $\begin{array}{l}\text { High D-dimer } \\
\text { group }(n=1974)\end{array}$ & $p$-value \\
\hline \multicolumn{6}{|l|}{ Demographics } \\
\hline Age, yrs & $62.2 \pm 11.3$ & $58.4 \pm 10.2$ & $62.9 \pm 10.5$ & $65.2 \pm 12.1$ & $<0.001$ \\
\hline male & $4117(69.5)$ & $1495(75.7)$ & $1343(68.0)$ & $1279(64.8)$ & $<0.001$ \\
\hline \multicolumn{6}{|l|}{ Medical history } \\
\hline History of Diabetes Mellitus & $1875(31.7)$ & $595(30.1)$ & $630(31.9)$ & $650(32.9)$ & 0.159 \\
\hline History of Hypertension & $3517(59.4)$ & $1116(56.5)$ & $1228(62.2)$ & $1173(59.4)$ & 0.001 \\
\hline History of Ml & $563(9.5)$ & $169(8.6)$ & $199(10.1)$ & $195(9.9)$ & 0.208 \\
\hline Dyslipidemia & $3992(67.4)$ & $1369(69.3)$ & $1324(67.1)$ & $1299(65.8)$ & 0.058 \\
\hline Prior $\mathrm{PCl}$ & $627(10.6)$ & $193(9.8)$ & $225(11.4)$ & $209(10.6)$ & 0.252 \\
\hline \multicolumn{6}{|l|}{ Clinical Presentation } \\
\hline SBP on admission, $\mathrm{mm} \mathrm{Hg}$ & $135.2 \pm 21.9$ & $134.9 \pm 21.1$ & $136.4 \pm 21.8$ & $134.3 \pm 22.9$ & 0.004 \\
\hline Heart rate on admission, bpm & $75.0 \pm 14.0$ & $74.0 \pm 12.8$ & $74.1 \pm 13.8$ & $76.7 \pm 15.2$ & $<0.001$ \\
\hline GRACE score & $129.4 \pm 36.7$ & $119.3 \pm 34.3$ & $128.2 \pm 34.4$ & $140.8 \pm 38.1$ & $<0.001$ \\
\hline \multicolumn{6}{|l|}{ Diagnosis on admission } \\
\hline STEMI & $2062(34.8)$ & $574(29.1)$ & $640(32.4)$ & $848(43.0)$ & $<0.001$ \\
\hline NSTEMI-ACS & $3861(65.2)$ & $1401(70.9)$ & $1334(67.6)$ & $1126(57.0)$ & \\
\hline \multicolumn{6}{|l|}{ Laboratory results on admission } \\
\hline D-dimer, ng/mL & $125(73,230)$ & $57(39,73)$ & $125(104,148)$ & $328(230,518)$ & $<0.001$ \\
\hline Fibrinogen, g/L & $3.28 \pm 0.82$ & $3.13 \pm 0.72$ & $3.27 \pm 0.76$ & $3.42 \pm 0.95$ & $<0.001$ \\
\hline Troponin-I, ng/mL & $0.41(0.01,11.78)$ & $0.9(0.01,7.14)$ & $0.21(0.01,6.81)$ & $1.43(0.03,22.85)$ & $<0.001$ \\
\hline Creatinine, umol/l & $72(61,85)$ & $70(60,82)$ & $72(61,83)$ & $75(61,91)$ & $<0.001$ \\
\hline Albumin, $g / L$ & $39.5 \pm 3.9$ & $40.5 \pm 3.6$ & $39.7 \pm 3.7$ & $38.3 \pm 3.9$ & $<0.001$ \\
\hline Hemoglobin, $g / L$ & $136.6 \pm 16.8$ & $141.1 \pm 15.1$ & $136.5 \pm 15.9$ & $132.3 \pm 18.1$ & $<0.001$ \\
\hline Leukocyte count $\left(\times 10^{9} / \mathrm{L}\right)$ & $8.30 \pm 2.99$ & $8.09 \pm 2.83$ & $8.17 \pm 2.84$ & $8.63 \pm 3.25$ & $<0.001$ \\
\hline Platelet count $\left(\times 10^{9} / \mathrm{L}\right)$ & $206.6 \pm 60.2$ & $206.1 \pm 57.1$ & $207.7 \pm 59.2$ & $206.0 \pm 64.1$ & 0.476 \\
\hline $\mathrm{BNP}, \mathrm{ng} / \mathrm{L}$ & $123(41,342)$ & $108(95,131)$ & $114(42,303)$ & $214(77,547)$ & $<0.001$ \\
\hline \multicolumn{6}{|c|}{ Percutaneous coronary intervention details } \\
\hline Left main disease & $527(8.9)$ & $141(7.1)$ & $179(9.1)$ & $207(10.5)$ & 0.001 \\
\hline Three-vessel disease & $1496(25.3)$ & $413(20.9)$ & $513(26.0)$ & $570(28.9)$ & $<0.001$ \\
\hline Use of Intra-aortic Balloon Pump & $190(3.2)$ & $36(1.8)$ & $57(2.9)$ & $97(4.9)$ & $<0.001$ \\
\hline TIMI flow grade $0 / 1$ on arrival & $4610(77.8)$ & $1539(77.9)$ & $1480(75.0)$ & $1591(80.6)$ & $<0.001$ \\
\hline TIMI flow grade 3 post $\mathrm{PCl}$ & 5899 (99.6) & $1973(99.9)$ & $1968(99.7)$ & $1958(99.2)$ & 0.001 \\
\hline In-hospital Mortality & $53(0.9)$ & $9(0.5)$ & $6(0.3)$ & $38(1.9)$ & $<0.001$ \\
\hline
\end{tabular}

MI Myocardial infarction, $P C I$ Percutaneous coronary intervention, SBP Systolic blood pressure, bpm Beats per minute, STEMI ST-segment elevation myocardial infarction, NSTEMI-ACS Non-ST-segment elevation myocardial infarction of acute coronary syndrome, BNP Brain natriuretic peptide 
Table 2 Effects of multiple variables on Clinical Outcomes in Univariate and Multivariate Analysis

\begin{tabular}{|c|c|c|c|c|c|c|}
\hline & \multicolumn{3}{|c|}{ Univariate Analysis } & \multicolumn{3}{|c|}{ Multivariate Analysis } \\
\hline & $\overline{\mathrm{OR}}$ & $95 \% \mathrm{Cl}$ & $p$ value & $\overline{\mathrm{OR}}$ & $95 \% \mathrm{Cl}$ & $p$ value \\
\hline GRACE score & 1.034 & $1.028-1.041$ & $<0.001$ & & & \\
\hline \multicolumn{7}{|l|}{ D-dimer as a continuous variable } \\
\hline D-dimer per $100 \mathrm{ng} / \mathrm{mL}$ increase & 1.069 & $1.046-1.093$ & $<0.001$ & 1.060 & $1.026-1.094$ & $<0.001^{\circ}$ \\
\hline \multicolumn{7}{|l|}{ D-dimer as a categories variable } \\
\hline Low D-dimer group & Reference & & $<0.001$ & Reference & & 0.003 \\
\hline Intermediate D-dimer group & 0.689 & $0.245-1.941$ & 0.481 & 0.668 & $0.176-2.532$ & 0.553 \\
\hline High D-dimer group & 4.362 & $2.104-9.044$ & $<0.001$ & 3.079 & $1.079-8.788$ & $0.036^{\mathrm{a}}$ \\
\hline
\end{tabular}

${ }^{a}$ Adjusted for age, History of Hypertension, Dyslipidemia, SBP on admission, Heart rate on admission, Diagnosis on admission, Troponin-I, fibrinogen, creatinine, Albumin, Hemoglobin, Leukocyte count, Platelet count, BNP, Left main disease, Three-vessel disease, Use of Intra-aortic Balloon Pump and TIMI flow grade 3 post $\mathrm{PCl}$;

an indicator of goodness-of-fit of each risk model and to assess the calibration ability of them [17]. The Brier scores of D-dimer, GRACE score and GRACE score + D-dimer were also calculated [18]. Lower Brier scores indicate better calibration [18]. We also used the absolute integrated discrimination improvement (IDI) and categoryfree net reclassification improvement (NRI) to evaluate improvements in risk predictions quantify [19]. All tests were two-sided, and the statistical significance was defined as $p<0.05$. All statistical analyses calculated by the Statistical Analysis System version 9.4 (SAS, SAS Institute Inc., Cary, North Carolina, USA).

\section{Results}

\section{Participants and baseline characteristics}

The flowchart represented the patient selection (Fig. 1). Five thousand nine hundred twenty-three patients with ACS undergoing PCI were included in the final study cohort. It was then divided into three groups: (1) Low D-dimer group [ $n=1975]$; (2) Intermediate D-dimer group [ $n=1974]$; (3) High D-dimer group [ $n=1974]$. Table 1 showed the clinical characteristics. High Ddimer group had significantly higher percentages of left main disease, females, three-vessel disease, STEMI, use of Intra-aortic Balloon Pump and TIMI grade $0 / 1$ on arrival, compared with Low and Intermediate D-dimer group. It also had a tendency towards increasing age, heart rate, GRACE score, fibrinogen, Troponin-I, creatinine, leukocyte count and BNP on admission. The percentage of prior hypertension was significantly higher in Intermediate D-dimer group. There was a significant trend of decreasing systolic blood pressure in High Ddimer group. High D-dimer group also had a trend to have a higher in-hospital mortality $(1.9 \%$ vs 0.5 and $0.3 \%, p<0.001$ ) (Table 1).

\section{Prognostic performance of D-dimer for the prognosis prediction}

When as a continuous variable, D-dimer significantly predicted in-hospital mortality in the univariate Logistic regression analysis (OR: 1.069, 95\% CI: 1.046-1.093, $p<$ 0.001 , for per $100 \mathrm{ng} / \mathrm{mL}$ increase) (Table 2). After adjusting for covariates, D-dimer was still independently associated with in-hospital mortality: an increased inhospital mortality risk of $6.0 \%$ for per $100 \mathrm{ng} / \mathrm{mL}$ increase in D-dimer concentration (OR: 1.060, 95\% CI: 1.026-1.094, $p<0.001$ ) (Table 2).

When categorized into three groups, D-dimer remained significantly predictive of in-hospital mortality (Table 2). In the univariate Logistic regression analysis, High D-dimer group had a substantially higher risk of in-hospital death (OR: 4.362, 95\% CI: 2.104-9.044, $p<$ 0.001 ), compared with Low and Intermediate D-dimer group (Table 2). In the multivariable Logistic regression analysis, High D-dimer group still had a significantly higher in-hospital mortality (OR: 3.079, 95\% CI: 1.0798.788, $p=0.036$ ) (Table 2).

\section{Prognostic performance of D-dimer, GRACE score and GRACE+ D-dimer for the prognosis prediction}

The C-statistic of D-dimer, GRACE score and GRACE score $+\mathrm{D}$-dimer for predicting in-hospital mortality

Table 3 GRACE, GRACE+ D-dimer and D-dimer performance for the prognosis prediction

\begin{tabular}{|c|c|c|c|c|c|c|c|}
\hline & \multicolumn{4}{|c|}{ Discrimination } & \multicolumn{2}{|l|}{ Calibration } & \multirow{2}{*}{$\begin{array}{l}\text { Precision } \\
\text { Brier Score }\end{array}$} \\
\hline & C-statistic & Standard error & $p$ value & $95 \% \mathrm{Cl}$ & HL $p$-Value & $\mathrm{R}^{2}$ & \\
\hline GRACE score & 0.842 & 0.0285 & $<0.001$ & $0.833-0.851$ & 0.733 & 0.192 & 0.0085 \\
\hline D-dimer & 0.719 & 0.0414 & $<0.001$ & $0.708-0.731$ & 0.002 & 0.037 & 0.0089 \\
\hline GRACE+ D-dimer & 0.851 & 0.0285 & $<0.001$ & $0.842-0.860$ & 0.503 & 0.208 & 0.0083 \\
\hline
\end{tabular}


were 0.719 (95\% CI 0.708 to 0.731 ), 0.842 (95\% CI 0.833 to 0.851 ) and 0.851 (95\% CI 0.842 to 0.860 ) (Table 3 and Fig. 2), respectively. The cut-off values for D-dimer was $212 \mathrm{ng} / \mathrm{mL}$ with a sensitivity of 0.698 and a specificity of 0.724 .

\section{Improvement of the prognostic performance of GRACE score combining D-dimer}

The Hosmer-Lemeshow $p$ value of the GRACE score was highest; the Nagelkerke- $\mathrm{R}^{2}$ of GRACE +D-dimer was highest; the Brier score of GRACE $+\mathrm{D}$-dimer was lowest (Table 3). The prognostic performance of GRACE + D-dimer was better than GRACE score (C-statistic: $z=$ 2.269, $p=0.023$; IDI: 0.016, $p=0.032$; NRI: 0.291, $p=$ 0.035 ) and D-dimer (C-statistic: $z=3.114, p=0.001$; IDI: $0.051, \quad p<0.001 ; \quad$ NRI: $0.928, \quad p<0.001)$, respectively (Table 4).

\section{Discussion}

This study focused on the association between D-dimer and in-hospital mortality in ACS patients undergoing PCI. It found that: (1) D-dimer could independently predict in-hospital mortality; (2) GRACE score + Ddimer got a better prognostic performance than GRACE score, and D-dimer could significantly improve the prognostic performance of GRACE score.

As a kind of soluble degradation product of crosslinked fibrin, D-dimer increased when coagulation was active, thrombin was generated, or fibrin was formed [5]. Pathophysiological factors associated with plasma Ddimer levels in coronary artery disease patients were studied. In stable coronary artery disease subjects, high D-dimer had a significant association with plaque necrosis, lipoprotein (a) and plaque calcium [6]. D-dimer level also independently predicted no-reflow in STEMI patients with primary PCI $[7,8]$. A CMR imaging study also found that high D-dimer on admission predicted larger myocardial infarct size, larger area at risk, and smaller myocardial salvage index in STEMI patients undergoing PCI [9]. Moreover, D-dimer might be associated with advanced myocardial injury [9]. Furthermore, the association between higher D-dimer and the clinical long-term adverse outcome other studies was also confirmed. Two studies focused on the role of D-dimer for predicting the prognosis in patients with STEMI receiving PCI $[13,14]$. They confirmed that D-dimer could independently predict the long-term mortality in those patients [13, 14]. Other studies also verified and extended this observation. High D-dimer was associated with long-term adverse outcome in stable coronary artery disease [10, 11], and ACS [12]. Our study also found that D-dimer was independently associated with inhospital mortality in patients with ACS receiving PCI.

Taken together, these findings had clinical value. It may be worth to monitor D-dimer in patients with ACS, which would identify the ACS patients at high risk.

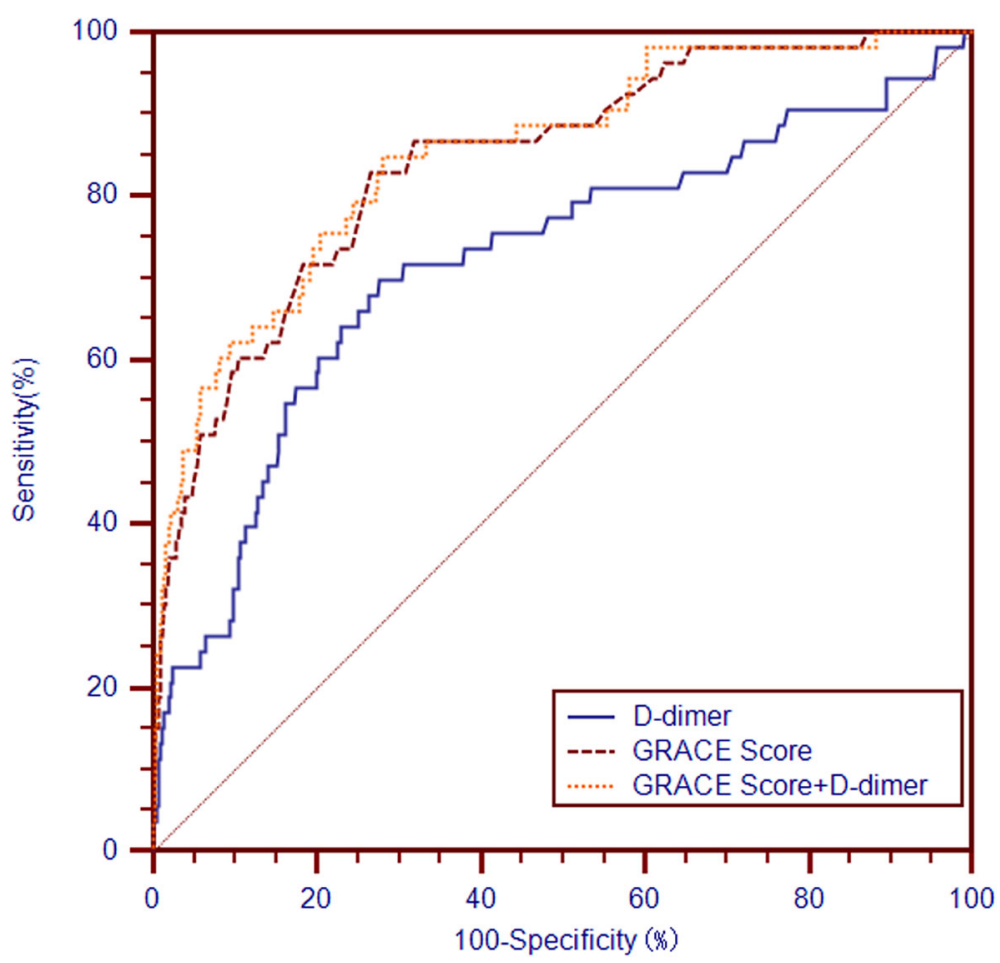

Fig. 2 Receiver operating characteristic curves of D-dimer, GRACE and GRACE+D-dimer for in-hospital death prediction 
Table 4 Comparisons of the predictive performance of GRACE, GRACE+ D-dimer and D-dimer for the prognosis prediction

\begin{tabular}{|c|c|c|c|c|c|c|}
\hline & z for C-statistic & $p$ for C-statistic & $\mathrm{NRI}$ & $p$ for NRI & $\mid \mathrm{IDI}$ & $p$ for IDI \\
\hline GRACE vs. D-dimer & 2.786 & 0.005 & 0.941 & $<0.001$ & 0.045 & 0.003 \\
\hline GRACE+ D-dimer vs. GRACE & 2.269 & 0.023 & 0.291 & 0.035 & 0.016 & 0.032 \\
\hline GRACE+ D-dimer vs. D-dimer & 3.144 & 0.001 & 0.928 & $<0.001$ & 0.051 & $<0.001$ \\
\hline
\end{tabular}

Moreover, the LIPID study presented evidence that Ddimer reflected an inflammatory state [11]. The studies also showed that anticoagulant and anti-inflammatory treatments could reduce ischemic events and venous thromboembolism [20-23]. So, high D-dimer patients may benefit from the anticoagulant and anti-inflammatory treatments. Such a strategy was tested in the Attenuation of D-dimer Using Vorapaxar to Target Inflammatory and Coagulation Endpoints (ADVICE) (NCT02394730) in patients with HIV. In the future, adequately powered randomized studies, targeting on attenuation of high $\mathrm{D}$ dimer in ACS patients, should be performed to obtain the conclusions.

The GRACE score is a widely recommended and important prognostic tool in patients with ACS [1-4]. It contains the main traditional risk factors. However, recently, more and more new risk factors, which were not contained by GRACE score, were studied. D-dimer was an important member of them [5]. This study found that GRACE score combining D-dimer showed good discrimination, calibration and precision. The prognostic performance of GRACE score combining D-dimer was also better than GRACE score. The prognostic performance of the GRACE score could be significantly improved by D-dimer. With the help of the new model (GRACE score combining D-dimer), more accurate assessment of the in-hospital mortality risk and better clinical decisions in patients with ACS will be made.

There were some limitations in this study. Firstly, it was a single-center, prospective and observational study. It was hard to completely adjust potential confounders and selection bias. Secondly, high-sensitivity C-reactive protein, other proinflammatory cytokines, or markers of oxidative stress were not included in this study. However, the LIPID study confirmed that, even after adjustment for high-sensitivity C-reactive protein, D-dimer was still a significant predictor [11]. Thirdly, the use of unfractionated heparin or low molecular weight heparin might affect D-dimer levels. Fourthly, some high-risk patients might be excluded because of the use of unfractionated heparin or LMWH, which might cause the potential selection bias. At last, data about the history of medication treatment that influenced coagulation and inflammatory, such as statin, was not complete. Patients with the high coagulation and inflammatory status could benefit from the statin use [24].

\section{Conclusion}

D-dimer was an independent predictor of in-hospital mortality in patients with ACS undergoing PCI. The prognostic performance of GRACE score combining Ddimer was better than the GRACE score. D-dimer could significantly improve the prognostic performance of the GRACE score.

\section{Supplementary information}

Supplementary information accompanies this paper at https://doi.org/10 1186/s12872-019-1239-4.

Additional file 1: Table S1. Effects of multiple variables on in-hospital

mortality in Univariate Analysis.

\section{Abbreviations}

ACS: Acute coronary syndrome; BNP: Brain natriuretic peptide; bpm: beats per minute; Cls: Confidence intervals; GRACE score: The Global Registry of Acute Coronary Events score; HL: The Hosmer-Lemeshow test; IDI: Integrated discrimination improvement; LMWH: Low molecular weight heparin; MI: Myocardial infarction; NRI: Net reclassification improvement; NSTEMIACS: Non-ST-segment elevation myocardial infarction of acute coronary syndrome; PCl: Percutaneous coronary intervention; PCl: Percutaneous coronary intervention;; SBP: Systolic blood pressure; STEMI: ST-segment elevation myocardial infarction

\section{Acknowledgements}

None.

Authors' contributions

ZS conceived and designed the experiments. TY, JS, DH, JW and YJ performed the experiments. TY analyzed the data and wrote the paper. ZJS revised the paper. All authors have reviewed and agreed on the contents of this paper. All authors read and approved the final manuscript.

\section{Funding}

This research project was supported by grants from the Social Development Research Program of Liaoning Province (2011225020). It funded the collection of data and a revision of the written English.

\section{Availability of data and materials}

The datasets used and/or analyzed during the current study are available from the corresponding author on reasonable request.

\section{Ethics approval and consent to participate}

This study complies with the Declaration of Helsinki, and Shengjing Hospital of China Medical University Research Ethics Committee approved the research protocol. Written informed consent was formally obtained from all participants.

Consent for publication

Not applicable.

Competing interests

The authors declare that they have no competing interests. 
Received: 18 February 2019 Accepted: 25 October 2019

Published online: 10 November 2019

\section{References}

1. O'gara PT, Kushner FG, Ascheim DD, et al. 2013 ACCF/AHA guideline for the management of ST-elevation myocardial infarction: a report of the American College of Cardiology Foundation/American Heart Association task force on practice guidelines. J Am Coll Cardiol. 2013;61(4):e78-140.

2. Amsterdam EA, Wenger NK, Brindis RG, et al. 2014 AHA/ACC guideline for the Management of Patients with non-ST-elevation acute coronary syndromes: a report of the American College of Cardiology/American Heart Association task force on practice guidelines. J Am Coll Cardiol. 2014;64(24): e139-228.

3. Roffi M, Patrono C, Collet JP, et al. 2015 ESC guidelines for the management of acute coronary syndromes in patients presenting without persistent STsegment elevation: task force for the Management of Acute Coronary Syndromes in patients presenting without persistent ST-segment elevation of the European Society of Cardiology (ESC). Eur Heart J. 2016;37(3):267-315.

4. Ibanez B, James S, Agewall S, et al. 2017 ESC guidelines for the management of acute myocardial infarction in patients presenting with STsegment elevation: the task force for the management of acute myocardial infarction in patients presenting with ST-segment elevation of the European Society of Cardiology (ESC). Eur Heart J. 2018;39(2):119-77.

5. Meade TW, Mellows S, Brozovic M, et al. Haemostatic function and ischaemic heart disease: principal results of the Northwick Park Heart Study. Lancet (London, England). 1986;2(8506):533-7.

6. Kothari H, Nguyen AT, Yang X, et al. Association of D-dimer with plaque characteristics and plasma biomarkers of oxidation-specific epitopes in stable subjects with coronary artery disease. J Cardiovasc Transl Res. 2018. https://doi.org/10.1007/s12265-018-9790-4.

7. Sarli B, Akpek M, Baktir AO, et al. Impact of D-dimer level on postinterventional coronary flow and in-hospital MACE in ST-segment elevation myocardial infarction. Herz. 2015:40(3):507-13.

8. Erkol A, Oduncu V, Turan B, et al. The value of plasma D-dimer level on admission in predicting no-reflow after primary percutaneous coronary intervention and long-term prognosis in patients with acute ST segment elevation myocardial infarction. J Thromb Thrombolysis. 2014:38(3):339-47.

9. Choi S, Jang WJ, Song YB, et al. D-dimer levels predict myocardial injury in ST-segment elevation myocardial infarction: a cardiac magnetic resonance imaging study. PLoS One. 2016;11(8):e0160955.

10. Naruse H, Ishii J, Takahashi $H$, et al. Prognostic value of combination of plasma D-dimer concentration and estimated glomerular filtration rate in predicting long-term mortality of patients with stable coronary artery disease. Circ J. 2017:81(10):1506-13.

11. Simes J, Robledo KP, White HD, et al. D-dimer predicts long-term causespecific mortality, cardiovascular events and Cancer in stable coronary heart disease patients: the LIPID study. Circulation. 2018. https://doi.org/10.1161/ circulationaha.117.029901.

12. Mjelva OR, Ponitz V, Brugger-Andersen T, Grundt H, Staines H, Nilsen DW. Long-term prognostic utility of pentraxin 3 and D-dimer as compared to highsensitivity C-reactive protein and B-type natriuretic peptide in suspected acute coronary syndrome. Eur J Prev Cardiol. 2016;23(11):1130-40.

13. Akgul O, Uyarel $H$, Pusuroglu $H$, et al. Predictive value of elevated D-dimer in patients undergoing primary angioplasty for ST elevation myocardial infarction. Blood Coagul Fibrinolysis. 2013;24(7):704-10.

14. Kikkert WJ, Claessen BE, Stone GW, et al. D-dimer levels predict ischemic and hemorrhagic outcomes after acute myocardial infarction: a HORIZONSAMI biomarker substudy. J Thromb Thrombolysis. 2014;37(2):155-64.

15. Cutlip DE, Windecker S, Mehran R, et al. Clinical end points in coronary stent trials: a case for standardized definitions. Circulation. 2007;115(17):2344-51.

16. Delong ER, Delong DM, Clarke-Pearson DL. Comparing the areas under two or more correlated receiver operating characteristic curves: a nonparametric approach. Biometrics. 1988;44(3):837-45.

17. Lemeshow S, Hosmer DW Jr. A review of goodness of fit statistics for use in the development of logistic regression models. Am J Epidemiol. 1982; 115(1):92-106

18. Redelmeier DA, Bloch DA, Hickam DH. Assessing predictive accuracy: how to compare brier scores. J Clin Epidemiol. 1991;44(11):1141-6.

19. Pencina MJ, D'agostino RB Sr, D'agostino RB Jr, Vasan RS. Evaluating the added predictive ability of a new marker: from area under the ROC curve to reclassification and beyond. Stat Med. 2008;27(2):157-72; discussion 207-112.
20. Oldgren J, Wallentin L, Alexander $\mathrm{JH}$, et al. New oral anticoagulants in addition to single or dual antiplatelet therapy after an acute coronary syndrome: a systematic review and meta-analysis. Eur Heart J. 2013;34(22):1670-80.

21. Castellucci LA, Cameron C, Le Gal G, et al. Efficacy and safety outcomes of oral anticoagulants and antiplatelet drugs in the secondary prevention of venous thromboembolism: systematic review and network meta-analysis. BMJ (Clinical research ed). 2013;347:f5133.

22. Ridker PM, Everett BM, Thuren T, et al. Antiinflammatory therapy with Canakinumab for atherosclerotic disease. N Engl J Med. 2017;377(12):1119-31.

23. Lembo G. From clinical observations to molecular mechanisms and back to patients: the successful circuit of the CANTOS study. Cardiovasc Res. 2018; 114(1):e3-5.

24. Ray KK, Cannon CP. Pathological changes in acute coronary syndromes: the role of statin therapy in the modulation of inflammation, endothelial function and coagulation. J Thromb Thrombolysis. 2004;18(2):89-101.

\section{Publisher's Note}

Springer Nature remains neutral with regard to jurisdictional claims in published maps and institutional affiliations.

Ready to submit your research? Choose BMC and benefit from:

- fast, convenient online submission

- thorough peer review by experienced researchers in your field

- rapid publication on acceptance

- support for research data, including large and complex data types

- gold Open Access which fosters wider collaboration and increased citations

- maximum visibility for your research: over $100 \mathrm{M}$ website views per year

At $\mathrm{BMC}$, research is always in progress.

Learn more biomedcentral.com/submissions 